\title{
Electronic and Plasmonic Materials Inside Microstructured Optical Fibers
}

\author{
Pier J. A. Sazio ${ }^{1 *}$, Adrian Amezcua-Correa ${ }^{1}$, Chris E. Finlayson ${ }^{1}$, John R. Hayes ${ }^{1}$, Thomas J. \\ Scheidemantel $^{2,3}$, Neil F. Baril ${ }^{2,4}$, Bryan R. Jackson ${ }^{2,4}$, Dong-Jin Won ${ }^{2,5}$, Feng Zhang, ${ }^{2,3}$, Elena R. \\ Margine $^{2,3}$, Venkatraman Gopalan ${ }^{2,5}$, Vincent H. Crespi ${ }^{2,3,5}$ and John V. Badding \\ 1) Optoelectronics Research Centre, University of Southampton, Highfield, Southampton SO17 1BJ, UK \\ 2) Materials Research Institute, Pennsylvania State University, USA \\ 3) Department of Physics, Pennsylvania State University, USA \\ 4) Department of Chemistry, Pennsylvania State University, USA \\ 5) Department of Materials Science and Engineering, Pennsylvania State University, USA \\ *Email: pjas@soton.ac.uk
}

\begin{abstract}
We report the growth of metal and crystalline semiconductor structures within holey fibers via a high-pressure microfluidic chemical vapour deposition process, to create novel photonic, electronic and plasmonic functionality inside the fiber waveguide. (C)2007 Optical Society of America

OCIS codes: (060.2280) Fiber design and fabrication; (160.6000) Semiconductors, including MQW; (230.4000)

Microstructure fabrication
\end{abstract}

Synthesis of materials inside templates is a powerful method of fabricating novel photonic and electronic nanostructured devices. We have realised that microstructured optical fibers (MOFs), which offer highly versatile engineering of the internal air-silica microstructure resulting in capillary holes with a wide range of shapes and sizes in precise periodic or aperiodic spatial configurations, are ideal microreactors for the chemical vapour deposition of functional materials such as metals and bulk crystalline semiconductors [1]. Materials inside MOFs can interact with waveguided electromagnetic radiation over extended length scales that are not possible with typical planar device geometries, thus allowing for the exploitation of light-matter interaction phenomena that are otherwise too weak to utilise on shorter length scales. The challenge of filling such MOF templates is their extreme aspect ratio, which requires very high pressures (10 to $100 \mathrm{MPa}$ ) to facilitate rapid mass transport through the fiber pores. Deposition within a "honeycomb" large air fraction fiber with 2 micron pores (Fig. 1A) forms smooth hexagonal germanium (Fig. 1B) and silicon (Fig. 1C) tubes. The resulting faceted tubes resemble lithographically patterned micro/nanostructures, yet are formed in a simple deposition process.

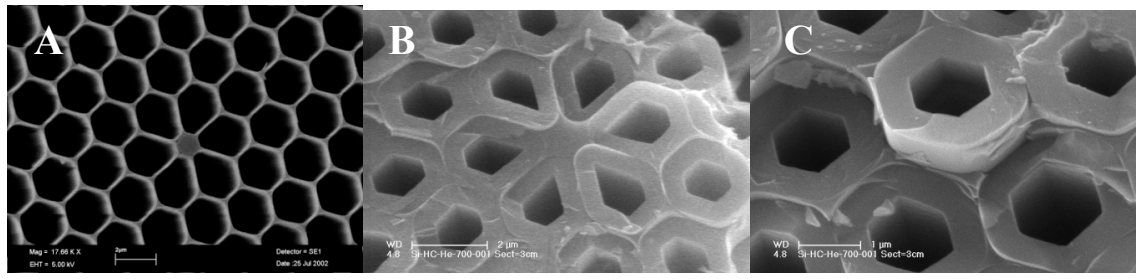

Figure 1. Unfilled silica "honeycomb" MOF template (A). Honeycomb after Ge deposition (B) and after Si deposition (C)

Hierarchical bottom-up organisation of nanomaterials into device configurations remains a central challenge in nanotechnology. Thus, the ability to engineer compositional complexity within optical fibers, provides an elegant and effective means of spatially organising functional materials to allow for co-operative photonic and electronic processes between them. Such devices are robust, inexpensive and can be seamlessly integrated into existing optical fiber infrastructure. This ability to fabricate optoelectronic fiber devices of unprecedented sophistication will be discussed, detailing some of our first generation demonstrator device configurations.

References

[1] P.J.A. Sazio et. al., "Microstructured Optical Fibers as High-Pressure Microfluidic Reactors", Science 311, 1583-1586 (2006). 